\title{
An exactly solvable model of the BCS-BEC crossover
}

\author{
J.N. Fuchs, A. Recati, W. Zwerger \\ Institute for Theoretical Physics, Universität Innsbruck, Technikerstrasse 25, A-6020 Innsbruck, Austria
}

(Dated: September 24, 2018)

\begin{abstract}
We discuss an integrable model of interacting Fermions in one dimension, that allows an exact description of the crossover from a BCS- to a Bose-like superfluid. This model bridges the GaudinYang model of attractive spin 1/2 Fermions to the Lieb-Liniger model of repulsive Bosons. Using a geometric resonance in the one-dimensional scattering length, the inverse coupling constant varies from $-\infty$ to $+\infty$ while the system evolves from a BCS-like state through a Tonks gas to a weakly interacting Bose gas of dimers. We study the ground state energy, the elementary density and spin excitations, and the correlation functions. An experimental realization with cold atoms of such a one-dimensional BCS-BEC crossover is proposed.
\end{abstract}

PACS numbers: 03.75.Ss, 74.20.Fg, 03.75.Hh

Starting with the historic controversy between Bardeen and Schafroth about the proper explanation of superconductivity [1], the crossover from a Bardeen-CooperSchrieffer (BCS) superfluid with Cooper pairs, whose size is much larger than the inter-particle spacing, to a BoseEinstein Condensate (BEC) of molecules composed of tightly bound Fermion pairs has been a basic issue in many-body physics [2]. Very recently, with the observation of molecular condensates near a Feshbach resonance in cold atomic Fermi gases [3], this problem has regained a lot of attention, since for the first time the crossover can be studied in detail. While there is general agreement on the fact that the evolution between the BCS and Boselimit is continuous, all existing theories of the crossover are approximate [4]. In particular, there is no reliable description of the most interesting regime near the Feshbach resonance, where the scattering length diverges. It is therefore of considerable interest to have an analytically soluble model of the BCS-BEC crossover. This is what we will provide in this letter for the particular case of one dimension (1D).

Cold gases allow to realize the BCS-BEC crossover by driving a mixture of two spin states with an attractive interaction through a Feshbach resonance, beyond which a bound state appears in the two particle problem in free space. On the BCS side of the crossover, pairs only exist in the many-body system due to the Pauli blocking of states below the Fermi energy. In one and also in two dimensions, the situation is quite different, however, because any purely attractive interaction produces a bound state already at the two particle level. In fact, contrary to the $3 \mathrm{D}$ case, its existence is both a necessary and sufficient condition for a BCS instability [5] . As will be shown below, an analog of the 3D crossover can be achieved in $1 \mathrm{D}$ by exploiting a confinement induced resonance (CIR) in a tight trap where the effective 1D scattering length exhibits a resonance caused by the mixing with a closed channel bound state in the trap [] $]$.

We start by considering the Hamiltonian of the Gaudin-Yang (GY) model [7] of a spin 1/2 Fermi gas interacting via a short range potential $g_{1} \delta(x)$ :

$$
H=-\frac{\hbar^{2}}{2 m} \sum_{i=1}^{N} \frac{\partial^{2}}{\partial x_{i}^{2}}+g_{1} \sum_{i<j} \delta\left(x_{i}-x_{j}\right)
$$

where $N$ is the total number of Fermions and $m$ their mass. The single dimensionless coupling constant is $\gamma \equiv m g_{1} / \hbar^{2} n$, where $n \equiv N / L$ is the $1 \mathrm{D}$ density. For attractive interactions, the Hamiltonian (1) describes a Luther-Emery liquid [8]. When $\gamma \rightarrow 0^{-}$, its ground state is a BCS-like state with Cooper pairs, whose size is much larger than the average inter-particle spacing [9]. The strong coupling regime with tightly bound molecules is now simply reached by increasing the magnitude of $\gamma$. The resulting Fermion pairs behave like a hard core Bose gas, or equivalently like 1D non-interacting Fermions [10]. In this manner, obviously, one never reaches a weakly interacting BEC as one of the limits of the standard BCS$\mathrm{BEC}$ crossover in $3 \mathrm{D}$. This is a consequence of the trivial fact that the two-body potential $g_{1} \delta(x)$ has a bound state only when $g_{1}<0$, but none when $g_{1}>0$. As a result, in the regime $g_{1}>0$, the ground state of the Hamiltonian (1) is that of repulsive Fermions and thus has nothing in common with a weakly interacting Bose gas of molecules.

In order to obtain a genuine BCS-BEC crossover in $1 \mathrm{D}$, it is necessary to exploit a geometric resonance in the $3 \mathrm{D}$ two-body scattering problem with a strong transverse confinement. For simplicity, we assume the Fermions to be confined in a waveguide with radial frequency $\omega_{\perp} / 2 \pi$ and oscillator length $a_{\perp} \equiv \sqrt{\hbar / m \omega_{\perp}}$. Moreover, we restrict ourselves to a truly $1 \mathrm{D}$ situation, where only the lowest transverse mode is occupied, requiring $\hbar \omega_{\perp}$ to be much larger than the Fermi energy $\epsilon_{F}$. As shown by Bergeman et al. [11], in such a situation there is always precisely one two-body bound state for the longitudinal motion with energy $\tilde{\epsilon}_{b}$, whatever the 3D scattering length $a$. Apart from this bound state, all the scattering properties are perfectly described by an effective 1D delta potential $g_{1} \delta(x)$ with strength $[\underline{\underline{6}}]$ :

$$
g_{1}=2 \hbar \omega_{\perp} a\left(1-A a / a_{\perp}\right)^{-1}
$$

As naively expected, an attractive 3D interaction $a<0$ 
implies a negative value of $g_{1}$, associated with a bound state whose energy $\epsilon_{b}=-m g_{1}^{2} / 4 \hbar^{2}$ coincides with the exact bound state energy $\tilde{\epsilon}_{b}$ in the limit $a / a_{\perp} \rightarrow 0$. Remarkably, $g_{1}$ and thus the binding energy $\epsilon_{b}$ remain finite at a Feshbach resonance $(a= \pm \infty)$. Entering the positive side $a>0$, however, the vanishing of the denominator at $a_{\perp} / a=A \simeq 1.0326$ [12] leads to a CIR, where $g_{1}$ jumps from $-\infty$ to $+\infty$ just as in a standard 3D Feshbach resonance, as discussed recently also in [13]. Now, for $g_{1}>0$, the short range potential $g_{1} \delta(x)$ no longer has a bound state, however it is still there in the $3 \mathrm{D}$ problem. Due to the condition $\hbar \omega_{\perp} \gg \epsilon_{F}$ (equivalently $\left(n a_{\perp}\right)^{2} \ll 1$ ), the true bound state energy at CIR, $\tilde{\epsilon}_{b}=-2 \hbar \omega_{\perp}$ [11], is much lower in energy than any other relevant scales and may formally be taken to minus infinity.

The 1D analog of a Feshbach resonance driven BCS$\mathrm{BEC}$ crossover in $3 \mathrm{D}$ is therefore described by a modified Gaudin-Yang model (11), where for positive $g_{1}$ the repulsive short range potential is supplemented by an additional bound state with energy $\epsilon_{b}=-\infty$. After crossing the CIR at $1 / \gamma=0$, the unbreakable Fermion pairs are described by a Lieb-Liniger (LL) model 14] of repulsive Bosons. On a formal level, the continuous evolution from an attractive Fermi to a repulsive Bose gas in one dimension is implicit in the Bethe Ansatz equations of the GY and LL models. The ground state for both $\gamma<0$ and $\gamma>0$ may be obtained from the solution of the equations:

$$
\begin{aligned}
\pi \rho(k) & =1+\int_{-K}^{K} \frac{d q}{n} \frac{\gamma \rho(q)}{\gamma^{2}+[(k-q) / n]^{2}} \\
\frac{E_{0}}{N} & =\frac{\epsilon_{b}}{2}+2 \int_{-K}^{K} \frac{d k}{n} \rho(k) \frac{\hbar^{2} k^{2}}{2 m}
\end{aligned}
$$

where the quasi-momenta distribution $\rho(k)$ is normalized as $\int d k \rho(k)=n / 2$ and $E_{0}$ is the ground state energy. For $\gamma<0$, they reduce to the GY equations of an attractive Fermi gas while for $\gamma>0$, they reduce to the LL equations of a gas of dimers [15]. This equivalence shows that in the whole regime $\gamma>0$, the density of Bosons is $n_{B}=n / 2$, their mass is $m_{B}=2 m$, their coupling $g_{B}$ is identified with the one of the LL model and $\gamma \equiv m g_{B} / \hbar^{2} n$. Since one is now dealing with dimers, the relation between the coupling constant $g_{B}$ and the experimentally accessible parameters $a$ and $a_{\perp}$ is no longer given by (2), however. It requires a solution of the dimer-dimer scattering problem in the presence of a transverse confinement. In the limit $a \ll a_{\perp}$, one can use the free space result for the effective scattering length between point like dimers $a_{3 B} \approx 0.6 a$ [16], leading to $g_{B} \approx 1.2 \hbar \omega_{\perp} a$. Close to resonance $\left(a_{\perp} / a \gtrsim A\right)$, a plausible ansatz is:

$$
g_{B}=\sqrt{2} \hbar \omega_{\perp} a\left(1-A a / a_{\perp}\right)^{-1}
$$

It follows by assuming that an equation of the form (2) holds also for dimers and the requirement that the resonances match, while $a_{\perp}$ is reduced by a factor $1 / \sqrt{2}$. Note that (4) corresponds to an effective dimer scattering length $a_{3 B}=a / \sqrt{2}$.

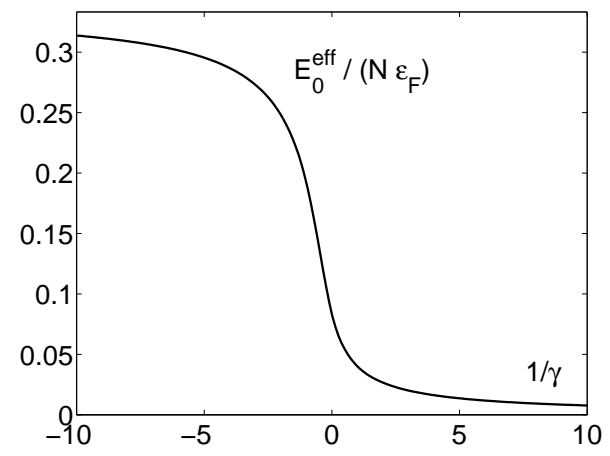

FIG. 1: Ground state effective energy per particle $E_{0}^{\text {eff }} / N=$ $E_{0} / N-\epsilon_{b} / 2$ [in units of the Fermi energy for the noninteracting gas $\left.\epsilon_{F}=\pi^{2} \hbar^{2} n^{2} / 8 m\right]$ as a function of $1 / \gamma$.

The qualitative physics of the modified Gaudin-Yang model is now simple to understand: when $1 / \gamma \rightarrow-\infty$ (BCS limit), the system consists of weakly bound Cooper pairs. The associated excitation gap is related to the spin sector, i.e. there is a finite gap between the singlet ground state and the first triplet excited state. In addition, there are gapless density fluctuations describing the Bogoliubov-Anderson mode of a neutral superfluid. At resonance, when $1 / \gamma=0$, the system is a Tonks gas [10] of tightly bound dimers [13]. It still exhibits sound modes with a linear spectrum, however the spin sector has disappeared because the spin gap diverges. On the positive side of the resonance, the system is an interacting Bose gas of tightly bound molecules. Its excitations are the standard Bogoliubov sound modes, whose velocity vanishes asymptotically in the weak coupling limit $1 / \gamma \rightarrow+\infty$ (BEC limit).

For quantitative results, we start with the ground state where the number of up and down spins is identical. Subtracting the bound state contribution, the relevant finite quantity to consider is $E_{0}^{e f f} \equiv E_{0}-N \epsilon_{b} / 2$, which we call the effective ground state energy. This quantity is obtained by numerically solving the Bethe Ansatz equations (3) and is plotted as a function of $1 / \gamma$ in Figure [1] Its asymptotic behavior in the BCS [9] and BEC-limit [14] and near the CIR is:

$$
\begin{aligned}
\frac{E_{0}^{e f f}}{N \epsilon_{F}} & \simeq \frac{1}{3}\left[1+\frac{6 \gamma}{\pi^{2}}-\frac{3 \gamma^{2}}{\pi^{4}} \log ^{2}(|\gamma|)+\cdots\right] ; 1 / \gamma \rightarrow-\infty \\
& \simeq \frac{1}{12}\left[1-\frac{1}{\gamma}+\frac{3}{4 \gamma^{2}}+\cdots\right] ; 1 / \gamma \rightarrow 0 \\
& \simeq \frac{\gamma}{\pi^{2}}\left[1-\frac{8 \sqrt{\gamma}}{3 \pi}+\cdots\right] ; 1 / \gamma \rightarrow+\infty
\end{aligned}
$$

where $\epsilon_{F} \equiv \pi^{2} \hbar^{2} n^{2} / 8 m$ is the Fermi energy for the noninteracting gas. We note that on resonance $E_{0}^{e f f}=$ $E_{0}^{e f f}\left(\gamma=0^{-}\right) / 4$

We now discuss the low energy elementary excitations. First consider the density excitations (the "charge sector") which are gapless phonons with dispersion relation 


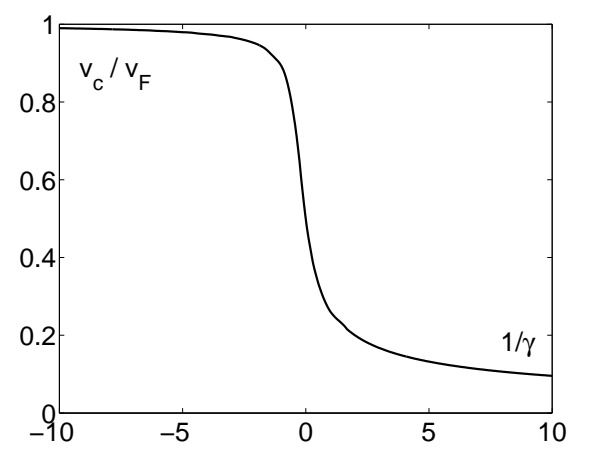

FIG. 2: Sound velocity $v_{c}$ [in units of the Fermi velocity for the non-interacting gas $\left.v_{F}=\pi \hbar n / 2 m\right]$ as a function of $1 / \gamma$.

$\omega=v_{c}|k|$, when $|k| \rightarrow 0$. An effective theory is provided by a Luttinger liquid description with charge velocity $v_{c}$ and correlation functions described by the Luttinger parameter $K_{c}$ [17]. When $\gamma<0, K_{c}^{(F)}$ describes fermionic correlation functions and is obtained from $K_{c}^{(F)}=v_{F} / v_{c}$ [18], where $v_{F} \equiv \pi \hbar n / 2 m$ is the Fermi velocity of the non-interacting gas. When $\gamma>0, K_{c}^{(B)}$ describes bosonic correlation functions and is given by $K_{c}^{(B)}=v_{F} / 2 v_{c}[18$. In order to determine these parameters, it is therefore enough to extract the sound velocity from the exact solution. This is done in the usual way by calculating the compressibility from the ground state energy [19]. Its asymptotic behavior in the BCS [9] and BEC-limit [14] and near the CIR is:

$$
\begin{aligned}
\frac{v_{c}}{v_{F}} & \simeq 1+\frac{\gamma}{\pi^{2}}+\frac{\gamma^{2}}{2 \pi^{4}}[\log (|\gamma|)-2]+\cdots ; 1 / \gamma \rightarrow-\infty \\
& \simeq \frac{1}{2}\left[1-\frac{1}{\gamma}+\frac{3}{4 \gamma^{2}}+\frac{3}{4 \gamma^{3}}+\cdots\right] ; 1 / \gamma \rightarrow 0 \\
& \simeq \frac{\sqrt{\gamma}}{\pi}\left[1-\frac{\sqrt{\gamma}}{2 \pi}+\cdots\right] ; 1 / \gamma \rightarrow+\infty
\end{aligned}
$$

Figure 2 shows $v_{c}$ as a function of $1 / \gamma$. Note that $K_{c}$ is always larger than one, which implies that the system is a 1D superfluid, as discussed, e.g., in [20].

The low energy spin excitations (the "spin sector") are described by a sine-Gordon model [19] with coupling parameter $\beta$. A recent renormalization group analysis of the sine-Gordon model [21, 22] shows that when $\gamma<0$ the system is driven to the strong coupling fixed point $\beta^{2}=4 \pi$, i.e., the spin Luttinger parameter $K_{s}[17]$ is equal to $1 / 2$. There it becomes equivalent to a noninteracting massive Thirring model [23], i.e., to a gas of massive relativistic fermions. The latter are called massive spinons and may be interpreted as a quantum soliton of the sine-Gordon model. They obey the relativistic dispersion relation:

$$
\omega=\sqrt{(\Delta / 2 \hbar)^{2}+\left(v_{s} k\right)^{2}}
$$

when $|k| \rightarrow 0$. Therefore, the low energy part of the spin sector is fully described by the spin velocity $v_{s}$ and the

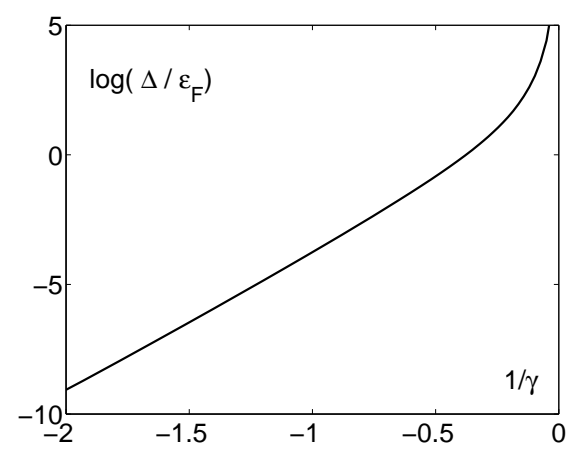

FIG. 3: Spin gap $\Delta$ [in units of the Fermi energy for the non-interacting gas $\left.\epsilon_{F}=\pi^{2} \hbar^{2} n^{2} / 8 m\right]$ as a function of $1 / \gamma$.

spin gap $\Delta$ or, equivalently, by the mass of the spinon $m_{s} \equiv \Delta /\left(2 v_{s}^{2}\right)$. The spin gap is also defined as the energy difference between the singlet ground state and the first triplet excited state. The spin velocity and the spin gap can be extracted from the spinon dispersion relation computed from the exact solution of the GY model. The spin gap has the following limiting behavior:

$$
\begin{aligned}
\frac{\Delta}{\epsilon_{F}} & \simeq \frac{16}{\pi} \sqrt{\frac{|\gamma|}{\pi}} e^{-\pi^{2} / 2|\gamma|}+\cdots ; 1 / \gamma \rightarrow-\infty \\
& \simeq \frac{2 \gamma^{2}}{\pi^{2}}\left[1-\frac{\pi^{2}}{4 \gamma^{2}}+\mathcal{O}\left(\gamma^{-4}\right)\right] ; 1 / \gamma \rightarrow 0^{-}
\end{aligned}
$$

where the BCS limit was already obtained in [9]. When $\gamma>0$, the spin gap is infinite in our model. Its behavior as a function of $1 / \gamma$ is plotted in Figure 3 . When $1 / \gamma<-1$, it behaves similarly to the gap computed from the BCS mean-field theory $\Delta_{B C S} \sim \epsilon_{F} \exp \left(-\pi^{2} / 2|\gamma|\right)$ and the dispersion relation is reminiscent of that of Bogoliubov quasiparticles, provided $\Delta_{B C S}$ is identified with $\Delta / 2[9]$. Near resonance, the spin gap is equal to the modulus of the two-body bound state energy $\left|\epsilon_{b}\right|=2 \gamma^{2} \epsilon_{F} / \pi^{2}$. The smooth crossover from Cooper pairs to molecules occurs when the size of a molecule is of the order of the average distance between particles $n^{-1}$, which happens for $\gamma \sim-2$.

The spin velocity can be computed with the bosonization approach in the weak coupling limit [17] and from the Bethe Ansatz equations in the strong coupling limit [9]. It turns out to be given by:

$$
\begin{aligned}
\frac{v_{s}}{v_{F}} & \simeq 1-\frac{\gamma}{\pi^{2}}+\cdots ; 1 / \gamma \rightarrow-\infty \\
& \simeq-\frac{\gamma}{\pi \sqrt{2}}\left[1-\frac{2}{\gamma}+\cdots\right] ; 1 / \gamma \rightarrow 0^{-}
\end{aligned}
$$

and is plotted as a function of $1 / \gamma$ in Figure 4 Spin correlation functions can be obtained from the knowledge of the spin gap $\Delta$ and of the charge Luttinger parameters $K_{c}$ and $v_{c}$, as shown in 24].

The above scenario for a BCS-BEC crossover can be realized in an experiment with ultra-cold gases confined 


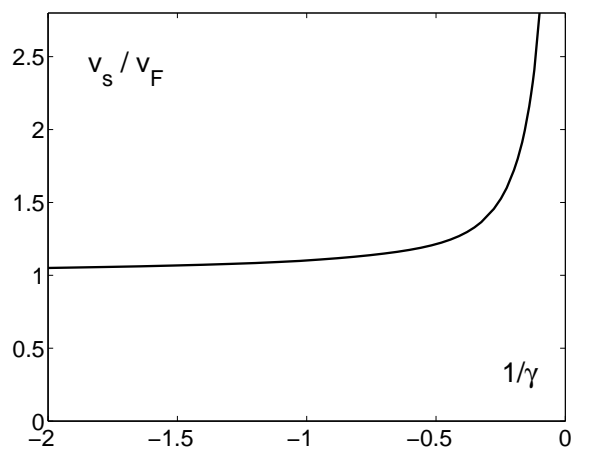

FIG. 4: Spin velocity $v_{s}$ [in units of the Fermi velocity for the non-interacting gas $\left.v_{F}=\pi \hbar n / 2 m\right]$ as a function of $1 / \gamma$.

in a quasi-1D geometry, e.g., ${ }^{6} \mathrm{Li}$ in an array of $1 \mathrm{D}$ tubes created with optical lattices [25] or on an atom chip [26]. In order to reach a $1 \mathrm{D}$ regime, the quantum of radial oscillation $\hbar \omega_{\perp}$ has to be much larger than (i) the quantum of axial oscillation $\hbar \omega_{\|}$, (ii) the Fermi energy $\epsilon_{F}$ and (iii) the thermal energy $k_{B} T$. Having a degenerate system imposes that the latter is also smaller than the Fermi energy. Note that condition (ii) is required for the validity of the model, as previously mentioned. Taking, e.g., the following values for radial $\omega_{\perp} / 2 \pi \sim 100 \mathrm{kHz}$ and axial $\omega_{\|} / 2 \pi \sim 200 \mathrm{~Hz}$ trap frequencies, number of particles (per tube) $N \sim 100$ [25] and temperature $T \sim 50 \mathrm{nK}[3$ ] allows to fulfill the previous requirements. To study the crossover in such a setting, the dimensionless coupling constant $\gamma$ needs to be tuned through a Feshbach resonance [3], giving rise to a CIR as discussed above. The experimental characterization of the different regimes can be done, for example, by measuring the axial collective modes in the trap. The ratio of the frequencies of the breathing and dipole modes 25] is 2 in both the BCS and CIR limit 13], and $\sqrt{3}$ in the BEC limit 27]. In addition, extending an available RF spectroscopy technique used to measure the molecular binding energy [28], it should be possible to extract information on the gap.

In conclusion, we have presented an exactly solvable model for a BCS-BEC crossover in 1D. The model may be realized experimentally by using the combination of a standard Feshbach- with a confinement-induced scattering resonance. In contrast to the $3 \mathrm{D}$ case, the exact solution allows to make quantitative predictions in the whole crossover regime. Our results show explicitly that the evolution from the BCS- to the BEC-limit is continuous, as expected on general grounds. Of course some of our results are specific for one dimensional systems, e.g., the pairs are unbreakable on the positive side of the resonance, which simplifies the description there to a purely bosonic model. Regarding the behavior of the sound velocity, however, the situation should be qualitatively similar to the 3D case. As such, our model provides a novel and experimentally accessible tool to address one of the long standing basic problems in many-body physics.

We thank W. Rantner and S. Cerrito for useful discussions and for carefully reading the manuscript. We also acknowledge discussions with P. Fedichev, P. Zoller, Z. Hadzibabic and C. Chin.

Note added. After this work was completed, we became aware of a related work of I.V. Tokatly [29].
[1] J. Matricon, G. Waysand, La Guerre du Froid: une Histoire de la Supraconductivité, Seuil (1994).

[2] D.M. Eagles, Phys. Rev. 186, 456 (1969); A.J. Leggett, J. Phys. C (Paris) 41, 7 (1980); P. Nozières, S. SchmittRink, J. Low Temp. Phys. 59, 195 (1985); M. Drechsler, W. Zwerger, Ann. Phys. (Germany) 1, 15 (1992).

[3] M. Greiner, C.A. Regal, D.S. Jin, Nature 426, 537 (2003); S. Jochim et al., Science 302, 2101 (2003); M.W. Zwierlein et al., Phys. Rev. Lett. 91, 250401 (2003).

[4] M. Randeria, in Bose-Einstein Condensation, ed. by A. Griffin, D.W. Snoke, S. Stringari, CUP (1995).

[5] M. Randeria, J.M. Duan, L.Y. Shieh, Phys. Rev. Lett. 62, 981 (1989).

[6] M. Olshanii, Phys. Rev. Lett. 81, 938 (1998).

[7] M. Gaudin, Phys. Lett. 24A, 55 (1967); C.N. Yang, Phys. Rev. Lett. 19, 1312 (1967).

[8] A. Luther, V.J. Emery, Phys. Rev. Lett. 33, 589 (1974).

[9] V.Ya. Krivnov, A.A. Ovchinnikov, Sov. Phys. JETP 40, 781 (1975).

[10] M. Girardeau, J. Math. Phys 1, 516 (1960).

[11] T. Bergeman, M.G. Moore, M. Olshanii, Phys. Rev. Lett. 91, 163201 (2003).

[12] Note the $\sqrt{2}$ difference in the definition of $a_{\perp}$ with [6, 11], which accounts for $A \equiv-\zeta(1 / 2,1) / \sqrt{2} \simeq 1.0326$.
[13] G.E. Astrakharchik, D. Blume, S. Giorgini, L.P. Pitaevskii, cond-mat/0312538

[14] E.H. Lieb, W. Liniger, Phys. Rev. 130, 1605 (1963); E.H. Lieb, Phys. Rev. 130, 1616 (1963).

[15] This was already noted by Gaudin 7].

[16] D.S. Petrov, C. Salomon, G.V. Shlyapnikov, cond-mat/0309010

[17] H.J. Schulz, "Fermi liquids and non-Fermi liquids" in Proceedings of Les Houches Summer School LXI, Elsevier (1995); cond-mat/9503150

[18] K.B. Efetov, A.I. Larkin, Sov. Phys. JETP 42, 390 (1976).

[19] H.J. Schulz, Phys. Rev. Lett. 64, 2831 (1990).

[20] C.L. Kane, M.P.A. Fisher, Phys. Rev. Lett. 68, 1220 (1992).

[21] S. Kehrein, Nucl. Phys. B[FS] 592, 512 (2001).

[22] The Hamiltonian's $S U(2)$ spin-invariance implies a particular trajectory in the RG parameter space, see [21].

[23] S. Coleman, Phys. Rev. D 11, 2088 (1975).

[24] E. Orignac, D. Poilblanc, Phys. Rev. B 68, 052504 (2003).

[25] H. Moritz et al., Phys. Rev. Lett. 91, 250402 (2003).

[26] J. Reichel, J.H. Thywissen, cond-mat/0310330

[27] C. Menotti, S. Stringari, Phys. Rev. A 66, 043610 (2002). 
[28] C.A. Regal, C. Ticknor, J.L. Bohn, D.S. Jin, Nature 424, 47 (2003); C. Chin, private communication and confer-

[29] I.V. Tokatly, cond-mat/0402276 ence in Levico 2004. 